\title{
Estudio de un Algoritmo Genético para la Administración Académica
}

\author{
Alvaro F. Salazar, Jesús F. López, Arturo Tavizón, María J. Araiza-Vázquez \\ Facultad de Contaduría Pública y Administración, Universidad Autónoma de Nuevo León, México \\ (e-mail: alvaro.salazargn@uanl.edu.mx; fabian.lopezpz@gmail.com; artavizons@gmail.com; araizav@gmail.com)
}

Recibido Dic. 4, 2018; Aceptado Feb. 12, 2019; Versión final Mar. 13, 2019, Publicado Ago. 2019

\section{Resumen}

El presente trabajo de investigación tiene como objetivo ayudar a evaluar la conveniencia del uso del método metaheurístico de algoritmos genéticos para la resolución de problemas de asignación de recursos académicos (docentes, aulas, horas de clase y asignaturas) en instituciones de educación superior. Esto se hace mediante el estudio del impacto de las características de tamaño del curso, disponibilidad de los docentes y la afinidad de docentes con materias en la resolución mediante algoritmos genéticos, comparando el tiempo requerido en encontrar una buena solución de asignación de los recursos y el óptimo alcanzado. El propósito de conocer los efectos estudiados en la aplicación del método de algoritmos genéticos fue logrado. Se encontraron indicios del impacto que la variación en cada uno de los parámetros tiene en el tiempo necesario para alcanzar una solución, lo cual ayudará a evaluar la conveniencia del uso de estas opciones de solución.

Palabras clave: administración académica; recursos académicos; asignación académica; algoritmos genéticos

\section{Study of a Genetic Algorithm for Academic Management}

\begin{abstract}
The aim of this research work is to help assessing the convenience of using the metaheuristic method of genetic algorithms to solve problems for the allocation of academic resources (teachers, classrooms, class hours and subjects in of higher education institutions. By studying the impact of size of the class, the availability of teachers and the affinity of teachers with subjects in the results achieved through genetic algorithms and comparing the time required to find a good solution to assign these resources and the optimum solution achieved. The purpose of knowing the effects studied in the application of the genetic algorithm's method was obtained. There were indications of the impact that the variation in each of the parameters has on the time necessary to reach a solution, which will help to evaluate the convenience of using these solution options.
\end{abstract}




\section{INTRODUCCIÓN}

En las escuelas de educación superior, se enfrenta periódicamente el problema administrativo de la asignación de recursos académicos, tales como: Docentes, Asignaturas, Horarios y Aulas. Esa toma de decisiones es de interés para las dependencias del referido tipo en cada inicio de período académico. Dicho proceso administrativo es, matemáticamente, complejo de resolver debido a la cantidad de combinaciones resultantes posibles, lo anterior ha dado origen a que se acuñe una taxonomía de problema identificada por algunos investigadores en lengua española como "Problema de Calendarización de horarios" (SanchezPartida et al, 2016), aunque en lengua inglesa existe una mayor cantidad de literatura especializada que aborda este tipo de problema y las diferentes combinaciones de los referidos recursos académicos denominando este tipo de problemas como "Class Teacher Timetabling Problem" o por sus siglas en inglés CTTP (Cataldo et al., 2017) relacionándose el presente trabajo por afinidad con las referidas líneas de investigación de estos y otros autores que abordan dichos estudios. Para afrontar este tipo de problemas, usualmente se recurre a repetir periódicamente plantillas de asignaciones anteriores, comprobadas empíricamente y sin una metodología científica; logrando de esta manera establecer dichas asignaciones. Esa forma de solución puede considerarse una Heurística, ya que consiste en buscar una solución aceptable mediante estimaciones y aproximaciones empíricas (RAE, 2012). Una adecuada asignación de los recursos académicos puede apoyar a la gestión de las instituciones educativas de nivel superior porque permite realizar un mejor manejo de la asignación de docentes adecuadamente calificados en las diferentes asignaturas, lo anterior debido a que se debe procurar que dichas asignaciones aprovechen el personal académico con que la institución cuenta y que sean capaces de alcanzar los mejores niveles de productividad posibles (Cancino y Márquez, 2015) y al mismo tiempo apoyar para tratar de eficientizar los costos en que se incurre al cumplir con ese propósito ya que se considera al costo como un factor importante en la determinación de la creación de valor que una escuela de educación superior puede aspirar a alcanzar (Zuniga-Jara et al., 2018)

Existen varias alternativas para abordar este tipo de problemas tal y como nos muestran los estudios de distintos autores, (Vergel 2017; Ramos y Bazzan 2015; Ahumada y Baloian 2014; Grunitzki et al., 2014; Tsutsui y Fujimoto 2011; Vásquez et al., 2010; Vannucci et al., 2006) algunas de ellas corresponden a la solución de este tipo de problemas mediante modelos matemáticos exactos que nos aseguran encontrar la mejor solución posible, si es que existe, teniendo como inconveniente que pueden llegar a ser muy tardados para ser prácticos y aplicables aunque se han logrado aplicar diversas estrategias que han tenido éxito en mejorar de forma competitiva dichas situaciones (Cataldo et al., 2017). Otra de dichas alternativas es la solución mediante métodos metaheurísticos, los cuales suelen ser una emulación de algún proceso de la naturaleza que nos permite encontrar una solución muy buena, sin asegurar que sea la mejor posible, pero en un lapso comparativamente corto y práctico. El uso de metaheurísticas ha demostrado obtener resultados particularmente buenos en problemas de programación de tablas de tiempos para universidades (Fong et al., 2014). Estas herramientas suelen ser complejas de implementar, sin embargo, actualmente se cuenta con algunas implementaciones comerciales disponibles para su implementación y aprovechamiento.

Algunos estudios internacionales han tratado de dar soluciones a esta problemática en su contexto particular, tal es el caso de Gómez et al (2005). Los cuales hicieron un estudio del problema instituyendo un modelo general del mismo, pero ajustándolo a su problema en particular, con el fin de implementar un algoritmo que les entregara soluciones cuasi optimas y en tiempos razonables. Otro estudio realizado en Uruguay por (Costabel, 2005), aplico la metaheurística en la asignación de horarios en la Universidad de República de Uruguay, se pretendía construir un motor capaz de apoyar la herramienta de software el cual incluye una combinación de metaheurísticas y algoritmos genéticos, búsqueda tabú y dentro de optimización por colonia de hormigas. Desafortunadamente se obtuvieron las peores soluciones, sin embargo, estudios más recientes como uno realizado en Perú (Cortez et al., 2010), proponen que el éxito a la solución de este tipo de algoritmos depende en gran medida a una agraciada definición de su función de evaluación y la codificación llevada a cabo del problema. Ellos establecieron un modelo que sirviera de apoyo a la generación de horarios, el cual definía las restricciones del problema y después se instituyó un boceto de la solución accediendo la adaptación de algoritmos genéticos. En el mismo sentido, el Politécnico di Milano (Dorigo y Stützle, 2019) presentaron resultados de una investigación que buscaba medir las posibilidades que ofrecían los algoritmos genéticos en la solución de problemas de asignación de horarios y la comparación con otras metaheurísticas, el desenlace que obtuvieron fue que los algoritmos genéticos producían mejores soluciones que el algoritmo de enfriamiento lento simulado, pero no eran tan buenos como los resultados del algoritmo búsqueda tabú, sin embargo, los algoritmos genéticos permitían flexibilidad para adaptarlos y resolver otros problemas de asignación de horarios.

Resulta oportuno mencionar que, a nivel nacional, en México, también se han realizado estudios afines; tal es el caso de un modelo de asignación de carga académicas usando algoritmos genéticos, capaces de encontrar la asignación óptima de clases, profesores y horarios en el Instituto Tecnológico de Nuevo Laredo, México (López y Johnston, 2007). Dicho modelo fue simplificado y adaptado para mostrar la relación grupo- 
materia, profesor y en Nuevo León (Johnston, 2000), aplicó algoritmos genéticos para la asignación de carga académica en instituciones de educación superior (IES) donde se tomaron en consideración diversos factores involucrados. Desarrollaron un prototipo funcional con un número muy limitado de restricciones que permitía darles valor de penalización, utilizando la minimización como función de evaluación.

Cada caso de aplicación de estas herramientas implica la resolución de un problema con diferentes magnitudes que pueden variar entre las diferentes instituciones educativas de acuerdo con las necesidades particulares de cada una. Al planteamiento de un problema de administración de los referidos recursos académicos se le denomina una instancia, la cual se puede describir mediante la variación en los denominados parámetros que presente (cantidad de recursos a combinar, compatibilidad entre ellos, entre otros). Actualmente se siguen realizando aproximaciones de solución a este tipo de problemas y sus variaciones con desarrollos personalizados de diversas metaheurísticas con un buen grado de éxito (Goh et al., 2017), motivados por estudios previos que han analizado propiamente la estructura de ese tipo de problemas de asignación de cursos (Burke et al., 2010). Sin embargo, pese al éxito reportado en esos casos, su principal desventaja es que es sumamente complicado pretender hacer un uso generalizado de las soluciones obtenidas, ya que suelen ser desarrollos ad-hoc para que puedan soportar las necesidades específicas de cada caso resuelto. Es por eso que el presente estudio se basa en la aplicación de uno de los referidos complementos comerciales y en la manera en que las características de la instancia afectan su desempeño. Específicamente nos referimos a la metaheurística denominada Algoritmos Genéticos que busca emular los procesos evolutivos representando las soluciones encontradas a través de códigos genéticos y aplicar procesos de selección natural procurando fomentar la evolución iterativa de las soluciones al desechar los organismos menos adaptados y preservar la aportación a su descendencia de los mejores candidatos (Holland, 1992).

El problema específico a abordar en este estudio es identificar de qué manera se ve afectado el desempeño de la metaheurística de algoritmos genéticos de acuerdo a las variaciones en tres de los parámetros con mayor ocurrencia en las diversas instancias, definiéndolos como: El tamaño de la instancia (La cantidad de aulas, docentes, horarios y asignaturas a combinar), la afinidad de los docentes con las diferentes asignaturas y la disponibilidad de los docentes o afinidad de estos con los horarios de clase. Lo anterior manteniendo un especial interés en dos factores principalmente: el tiempo que transcurre para encontrar la mejor solución alcanzable y la optimalidad de dicha solución con respecto al máximo posible.

Se pretenden comprobar las siguientes hipótesis de estudio: $\mathrm{H}_{1}$ : Tamaño Bajo $\neq$ Tamaño Medio $\neq$ Tamaño Alto; $\mathrm{H}_{2}$ : Afinidad Maestro Materia Baja $\neq$ Afinidad Maestro Materia Media $\neq$ Afinidad Maestro Materia Alta; $y$ $\mathrm{H}_{3}$ : Afinidad Maestro Horario Baja $\neq$ Afinidad Maestro Horario Media $\neq$ Afinidad Maestro Horario Alta. Estas, a su vez, para fines de estudio descompondremos en las siguientes hipótesis de trabajo que confrontarán cada una de las variaciones dentro de los parámetros respecto a su impacto en los resultados obtenidos a través de la referida metaheurística: $\mathrm{H}_{1}$ : TamañoBajo $\neq$ Tamaño Medio; $\mathrm{H}_{2}$ : Tamaño Medio $\neq$ Tamaño Alto; $\mathrm{H}_{3}$ : Tamaño Bajo $\neq$ Tamaño Alto; $\mathrm{H}_{4}$ : Afinidad Maestro Materia Baja $\neq$ Afinidad Maestro Materia Media; $\mathrm{H}_{5}$ : Afinidad Maestro Materia Media $\neq$ Afinidad Maestro Materia Alta; $\mathrm{H}_{6}$ : Afinidad Maestro Materia Baja $\neq$ Afinidad Maestro Materia Alta; $\mathrm{H}_{7}$ : Afinidad Maestro Horario Baja $\neq$ Afinidad Maestro Horario Media; $\mathrm{H}_{8}$ : Afinidad Maestro Horario Media $\neq$ Afinidad Maestro Horario Alta; $y$ Hs: Afinidad Maestro Horario Baja $\neq$ Afinidad Maestro Horario Alta]

\section{METODOLOGÍA}

Se definieron escenarios simulados en concordancia con modelos planteados como representativos en la literatura académica especializada (Bellio et al, 2016) que permitieran contrastar mediante la vía experimental los efectos de variaciones en cada uno de los tres parámetros estudiados en tres niveles diferentes para cada uno de ellos en las diferentes permutaciones posibles. A saber, los parámetros estudiados fueron: El tamaño de la instancia, la afinidad de los docentes con las asignaturas a impartir y la disponibilidad horaria de los docentes. Los diferentes estímulos aplicados en los parámetros pueden apreciarse en la tabla 1, de la cual se considera pertinente aclarar que el parámetro correspondiente al tamaño de la instancia se expresa en cuanto a la cantidad de posibles combinaciones entre los conjuntos de elementos a asignar: a) Cantidad de docentes, b) Cantidad de materias, c) Cantidad de aulas y d) Cantidad de horas/clase a asignar. El proceso metodológico se presenta sintetizado en la figura 1 con sus diferentes procesos y seguimientos correspondientes.

Lo anterior tomando como base que el nivel bajo de tamaño de la instancia está dado por el resultado de calcular las referidas combinaciones considerando: 15 docentes, 15 aulas, 10 materias, 10 horas de clase. Esto es: $(15)(15)(10)(10)=22,500$. Por otra parte, los parámetros de Afinidad de los docentes con las asignaturas y la disponibilidad horaria de los mismos docentes, la variación entre cada nivel adyacente se estableció en $10 \%$, estableciendo el nivel medio de ambos parámetros en $50 \%$. 


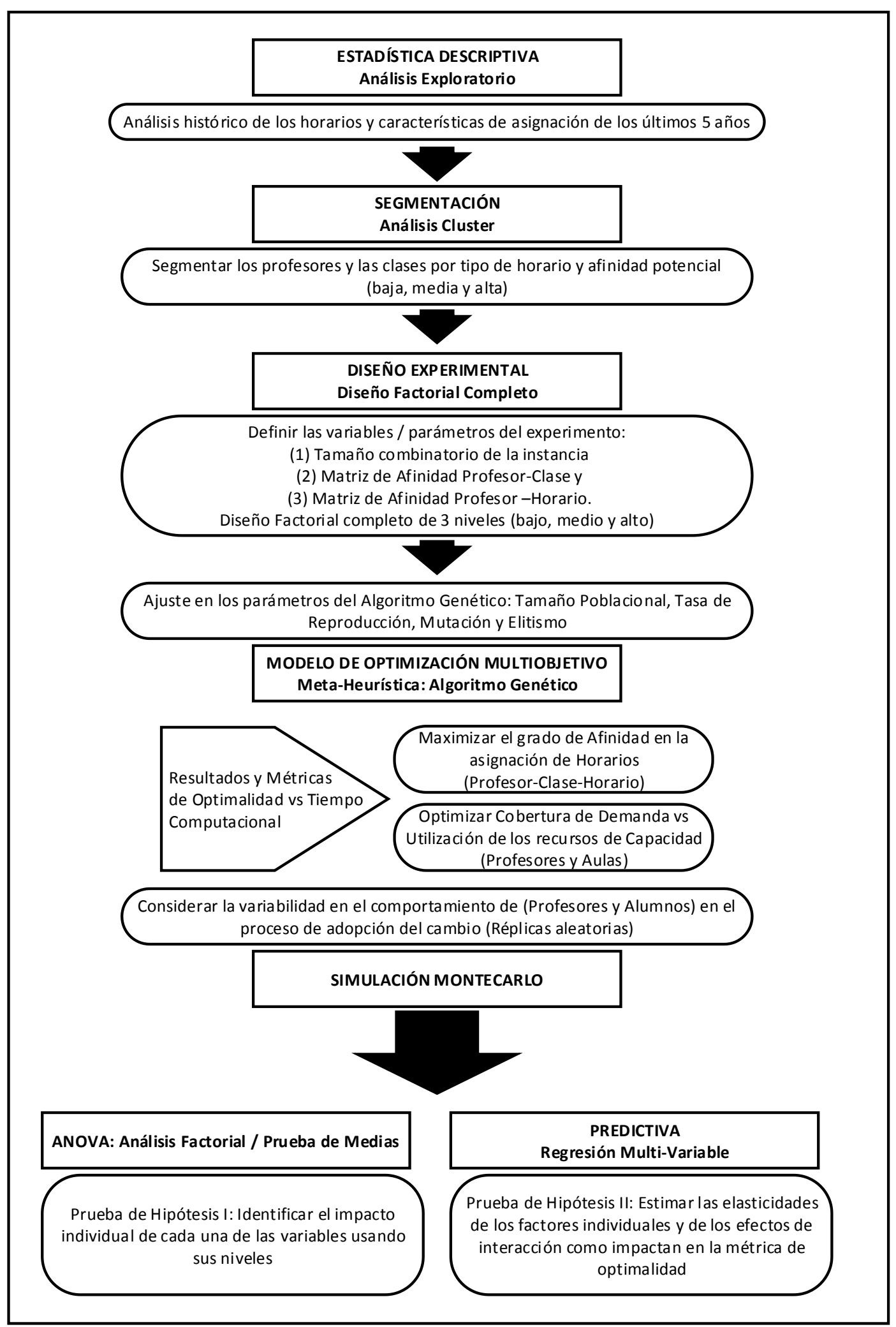

Fig. 1: Esquema del proceso metodológico

Tabla 1: Proporción de variación en los niveles para los parámetros estudiados

\begin{tabular}{|l|c|c|c|}
\cline { 2 - 4 } \multicolumn{1}{c|}{} & $\begin{array}{c}\text { Tamaño (Combinaciones } \\
\text { posibles) }\end{array}$ & Afinidad & Disponibilidad \\
\hline Bajo & 22,500 & $40 \%$ & $40 \%$ \\
\hline Medio & 360,000 & $50 \%$ & $50 \%$ \\
\hline Alto & $5,760,000$ & $60 \%$ & $60 \%$ \\
\hline
\end{tabular}


De acuerdo con lo anterior la variación del parámetro de tamaño de la instancia fue tal que cada incremento corresponde al doble del tamaño anterior; esto es: teniendo como nivel base el nivel bajo, el nivel medio corresponde al doble de tamaño en cada una de sus cuatro dimensiones a considerar y a su vez el nivel superior del tamaño de la instancia corresponde al doble de tamaño que el corresponde al nivel medio. En el caso del parámetro de Tamaño de la instancia el cambio entre niveles tiene una proporción mayor, ya que en cada nivel se dobló la cantidad de cada uno de los cuatro elementos a combinar (Docentes, aulas, materias y horas de clase) Ocasionando un crecimiento exponencial en la variación de este parámetro evidenciado en la tabla 1 resultando en que el nivel medio posee 16 veces más combinaciones que el nivel bajo y que el nivel alto posee 16 veces más combinaciones que el nivel medio y 256 veces más que el nivel bajo.

Por lo que corresponde a los parámetros de Afinidad de los docentes con las asignaturas se determinó considerando una escala de cuatro valores posibles que abarca desde 1 (Pésimamente calificado) hasta 4 (Altamente calificado) y se consideró un cálculo ponderado estimado mediante la presencia de cada valor por su aportación sumativa al total de la afinidad en la matriz generada para tal efecto al combinar los docentes con las materias. El cálculo de la disponibilidad horaria de los mismos docentes siguió un concepto similar, excepto por ser dicotómico ya que el docente podía manifestar su disposición para dar clase en ese horario (Estableciendo la conjunción en el valor de 1), o bien indicar que no desearía impartir clase en ese horario (Obteniendo esa combinación el valor de 0). Por lo tanto, para estos dos últimos parámetros la variación entre cada nivel adyacente se estableció en $10 \%$, estableciendo el nivel medio de ambos parámetros en $50 \%$.

Una vez determinadas estas variaciones se estableció un modelo matemático de optimización en Microsoft Excel 2016 y para resolverlo se eligió el complemento de Excel denominado Evolver de la empresa Palisade el cual proporciona la opción de resolver problemas de optimización mediante la metaheurística de Algoritmos genéticos y encargándose de manejar los procesos de bajo nivel de la metaheurística, permitiendo así una manera más simple de aplicarla. El equipo utilizado para realizar la ejecución del presente estudio contó con la siguiente configuración: a) Procesador Intel Core i5-3210M a 2.49GHz, b) Memoria RAM de seis Gigabytes y c) Sistema operativo Windows 8.1 de 64 bits.

\section{RESULTADOS Y DISCUSIÓN}

Al resolver mediante la referida metaheurística los diferentes escenarios del experimento, se obtuvieron los datos mostrados en la tabla 2, en la cual se puede apreciar para cada escenario de combinación de nivel de estímulo la optimalidad alcanzada y el tiempo computacional expresado en segundos que fue necesario para alcanzar el resultado obtenido.

Tabla 2: Listado de las diferentes combinaciones de variaciones en los parámetros a estudiar en el experimento

\begin{tabular}{|c|c|c|c|c|c|}
\hline Escenario & $\begin{array}{l}\text { Tamaño } \\
\text { Instancia }\end{array}$ & $\begin{array}{c}\text { Afinidad } \\
\text { Maestro/Materia }\end{array}$ & $\begin{array}{c}\text { Afinidad } \\
\text { Maestro/Hora }\end{array}$ & $\begin{array}{l}\text { Optimalidad } \\
\text { Alcanzada }\end{array}$ & $\begin{array}{c}\text { Tiempo computacional } \\
\text { (Segundos) }\end{array}$ \\
\hline 1 & Bajo & Bajo & Bajo & $97.14 \%$ & 51 \\
\hline 2 & Bajo & Bajo & Medio & $97.14 \%$ & 287 \\
\hline 3 & Bajo & Bajo & Alto & $98.10 \%$ & 96 \\
\hline 4 & Bajo & Medio & Bajo & $99.05 \%$ & 77 \\
\hline 5 & Bajo & Medio & Medio & $99.05 \%$ & 43 \\
\hline 6 & Bajo & Medio & Alto & $99.05 \%$ & 37 \\
\hline 7 & Bajo & Alto & Bajo & $99.05 \%$ & 108 \\
\hline 8 & Bajo & Alto & Medio & $100.00 \%$ & 40 \\
\hline 9 & Bajo & Alto & Alto & $100.00 \%$ & 33 \\
\hline 10 & Medio & Bajo & Bajo & $84.76 \%$ & 980 \\
\hline 11 & Medio & Bajo & Medio & $90.00 \%$ & 1840 \\
\hline 12 & Medio & Bajo & Alto & $87.62 \%$ & 1190 \\
\hline 13 & Medio & Medio & Bajo & $90.95 \%$ & 914 \\
\hline 14 & Medio & Medio & Medio & $89.05 \%$ & 1052 \\
\hline
\end{tabular}


Tabla 2 (continuación)

\begin{tabular}{|c|c|c|c|c|c|}
\hline Escenario & $\begin{array}{c}\text { Tamaño } \\
\text { Instancia }\end{array}$ & $\begin{array}{c}\text { Afinidad } \\
\text { Maestro/Materia }\end{array}$ & $\begin{array}{c}\text { Afinidad } \\
\text { Maestro/Hora }\end{array}$ & $\begin{array}{c}\text { Optimalidad } \\
\text { Alcanzada }\end{array}$ & $\begin{array}{c}\text { Tiempo computacional } \\
\text { (Segundos) }\end{array}$ \\
\hline 15 & Medio & Medio & Alto & $90.00 \%$ & 1007 \\
\hline 16 & Medio & Alto & Bajo & $99.05 \%$ & 991 \\
\hline 17 & Medio & Alto & Medio & $99.05 \%$ & 713 \\
\hline 18 & Medio & Alto & Alto & $99.05 \%$ & 5539 \\
\hline 19 & Alto & Bajo & Bajo & $82.62 \%$ & 5442 \\
\hline 20 & Alto & Bajo & Medio & $76.90 \%$ & 4774 \\
\hline 21 & Alto & Bajo & Alto & $82.85 \%$ & 4881 \\
\hline 22 & Alto & Medio & Bajo & $82.85 \%$ & 1004 \\
\hline 23 & Alto & Medio & Medio & $84.75 \%$ & 4913 \\
\hline 24 & Alto & Medio & Alto & $87.85 \%$ & 6182 \\
\hline 25 & Alto & Alto & Bajo & $90.94 \%$ & 2463 \\
\hline 26 & Alto & Alto & Medio & $87.84 \%$ & 5071 \\
\hline 27 & Alto & Alto & Alto & $94.52 \%$ & 706 \\
\hline
\end{tabular}

Con respecto al dato de optimalidad se consideró una cota superior teórica consistente en que todos los docentes tuvieran asignadas materias en las que son altamente competentes y en un horario deseado por el referido maestro por lo que la optimalidad alcanzada corresponde al porcentaje de puntaje obtenido con respecto al resultado que se obtendría al alcanzar la cota teórica superior ya indicada. Con los datos recopilados, se procedió a realizar las pruebas de hipótesis correspondientes mediante la distribución t debido a la cantidad de elementos de la muestra trabajada, obteniéndose los resultados mostrados en la tabla 3 para cada una de las hipótesis comprobadas $(\mathrm{H} 1, \mathrm{H} 2$ y H3) y resaltándose estas por estar señaladas mediante un carácter de asterisco, las cuales en este caso fueron aquellas que contrastan los grupos en función del tamaño de la instancia. Se aclara que los parámetros se expresan de acuerdo al siguiente formato Par.Nivel donde Par representa la abreviatura del parámetro (Tam: Tamaño de la instancia, Afin: Afinidad de los maestros con las asignaturas y Disp: Disponibilidad horaria de los maestros); así también la partícula Nivel representa el nivel del parámetro, ejemplo: Tam.Bajo indica el caso de considerar el tamaño bajo de la instancia

Tabla 3: Resultados de las pruebas t de hipótesis con dos muestras asumiendo varianzas desiguales con respecto al parámetro de Tamaño de la instancia mostrando las hipótesis aceptadas

\begin{tabular}{|c|c|c|c|c|c|}
\hline *H1 & Tam.Bajo & Tam.Medio & & Tam.Bajo & Tam.Medio \\
\hline & & & Media & 85.77778 & 1043.667 \\
\hline & & & t Calculada & -8.31534 & \\
\hline * $\mathrm{H} 2$ & Tam.Medio & Tam.Alto & & Tam.Medio & Tam.Alto \\
\hline & & & Media & 1043.667 & 4474.333 \\
\hline & & & t Calculada & -6.09882 & \\
\hline *H3 & Tam.Bajo & Tam.Alto & & Tam.Bajo & Tam.Alto \\
\hline & & & Media & 85.77778 & 4474.333 \\
\hline & & & t Calculada & -7.9519 & \\
\hline
\end{tabular}

El resto de las hipótesis $(\mathrm{H} 4$ a $\mathrm{H} 6$ para el parámetro de la afinidad del docente con la materia y $\mathrm{H} 7$ a $\mathrm{H} 9$ para el parámetro de la disponibilidad de horario) no lograron ser comprobadas mediante la prueba T de hipótesis, siendo esto evidente por los valores obtenidos para el valor de t calculada, lo cual se presenta en la tabla 4 para cada una de las hipótesis evaluadas. 
Tabla 4: Resultados de las pruebas t de hipótesis con dos muestras asumiendo varianzas desiguales con respecto al parámetro de Afinidad del docente con la materia mostrando las hipótesis rechazadas

\begin{tabular}{|c|c|c|c|c|c|}
\hline $\mathrm{H} 4$ & Afin.Bajo & Afin.Medio & & Afin.Bajo & Afin.Medio \\
\hline & & & Media & 2244.333333 & 1547.555556 \\
\hline & & & t Calculada & 0.687340808 & \\
\hline \multirow[t]{3}{*}{$\mathrm{H} 5$} & Afin.Medio & Afin.Alto & & Afin.Medio & Afin.Alto \\
\hline & & & Media & 1547.555556 & 1811.888889 \\
\hline & & & t Calculada & -0.26281 & \\
\hline \multirow{5}{*}{$\mathrm{H} 6$} & & & & & \\
\hline & Afin.Bajo & Afin.Alto & & Afin.Bajo & Afin.Alto \\
\hline & & & Media & 2244.333333 & 1811.888889 \\
\hline & & & t Calculada & 0.395532916 & \\
\hline & & & & & \\
\hline \multirow[t]{4}{*}{$\mathrm{H} 7$} & Disp.Bajo & Disp.Medio & & Disp.Bajo & Disp.Medio \\
\hline & & & Media & 2191.444444 & 1431.555556 \\
\hline & & & t Calculada & 0.741428055 & \\
\hline & & & & & \\
\hline \multirow[t]{3}{*}{$\mathrm{H} 8$} & Disp.Medio & Disp.Alto & & Disp.Medio & Disp.Alto \\
\hline & & & Media & 1431.55556 & 1980.77778 \\
\hline & & & t Calculada & -0.58435 & \\
\hline \multirow[t]{3}{*}{ H9 } & Disp.Bajo & Disp.Alto & & Disp.Bajo & Disp.Alto \\
\hline & & & Media & 2191.444444 & 1980.777778 \\
\hline & & & t Calculada & 0.18579133 & \\
\hline
\end{tabular}

Con la intención de obtener mayor información respecto a cómo los parámetros de las instancias afectan los resultados obtenidos se procedió a realizar dos análisis de regresión lineal con el propósito de identificar algún nivel de significancia posible de los tres parámetros de la instancia con respecto a dos variables dependientes: el tiempo y la optimalidad. En el primero de ellos, mostrado en la tabla 5, se considera a la variable dependiente como el tiempo requerido expresado en segundos para obtener la mejor solución posible expresada en segundos, y a los tres parámetros ajustados en cada escenario como las variables independientes de acuerdo a los niveles de estímulo establecidos.

Tabla 5: Resultado del análisis de regresión considerando al tiempo como la variable explicada

Variables introducidas/eliminadas considerando como variable dependiente el tiempo computacional expresado en segundos

\begin{tabular}{|c|c|c|c|c|c|}
\hline & Modelo & $\begin{array}{c}\text { Variables } \\
\text { introducidas }\end{array}$ & Variables eliminadas & \multicolumn{2}{|c|}{ Método } \\
\cline { 2 - 5 } & 1 & Tamañolnst & $\begin{array}{c}\text { Por pasos (criterio: Prob. } \\
\text { de F para entrar }<=.050, \\
\text { Prob. de F para salir }>= \\
\text {.100). }\end{array}$ \\
\hline \multicolumn{7}{|c|}{ Resumen del modelo considerando como variable predictora el tamaño de la instancia } \\
\hline \\
\hline Modelo & $\mathrm{R}$ & $\mathrm{R}$ cuadrado & $\begin{array}{c}\text { R cuadrada } \\
\text { corregida }\end{array}$ & $\begin{array}{c}\text { Error típico } \\
\text { de la } \\
\text { estimación }\end{array}$ \\
\hline \\
1 & $.855^{\mathrm{a}}$ & .730 & .720 & 1131.472 \\
\hline
\end{tabular}


Tabla 5 (continuación)

\begin{tabular}{|c|c|c|c|c|c|c|}
\hline \multicolumn{7}{|c|}{ Coeficientes considerando como variable dependiente el tiempo computacional expresado en segundos } \\
\hline \multirow{2}{*}{\multicolumn{2}{|c|}{ Modelo }} & \multicolumn{2}{|c|}{ Coeficientes no estandarizados } & \multirow{3}{*}{$\begin{array}{c}\begin{array}{c}\text { Coeficientes } \\
\text { tipificados }\end{array} \\
\text { Beta } \\
\end{array}$} & \multirow[t]{2}{*}{$\mathrm{t}$} & \multirow[t]{2}{*}{ Sig. } \\
\hline & & $\mathrm{B}$ & Error típico. & & & \\
\hline \multirow{2}{*}{1} & (Constante) & -2520.630 & 576.117 & & -4.375 & .000 \\
\hline & Tamañolnst & 2194.278 & 266.690 & .855 & 8.228 & .000 \\
\hline \multicolumn{7}{|c|}{$\begin{array}{l}\text { Variables excluidas considerando como variable dependiente el tiempo computacional y como variable } \\
\text { predictora el tamaño de la instancia }\end{array}$} \\
\hline \multirow{2}{*}{\multicolumn{2}{|c|}{ Modelo }} & Beta dentro & $\mathrm{t}$ & Sig. & Correlación & $\begin{array}{l}\text { Estadísticos } \\
\text { colinealidad }\end{array}$ \\
\hline & & & & & & Tolerancia \\
\hline \multirow{2}{*}{1} & Afinidad & $-.084^{\mathrm{b}}$ & -.805 & .429 & -.162 & 1.000 \\
\hline & Disponibilidad & $-.041^{\mathrm{b}}$ & -.388 & .701 & -.079 & 1.000 \\
\hline
\end{tabular}

En el siguiente análisis mostrado en la tabla 6 , se consideró a la variable dependiente como el porcentaje de optimalidad alcanzado y a los mismos tres parámetros del caso anterior como las variables independientes de acuerdo a los ya referidos niveles de estímulo establecidos. Ambos estudios de regresión lineal se realizaron utilizando el software SPSS versión 20 en el mismo equipo especificado en la sección de metodología y en el que se ejecutó la totalidad de las mediciones que abarca el presente trabajo de investigación.

Tabla 6: Resultado del análisis de regresión considerando la optimalidad como la variable explicada

Variables introducidas/eliminadas considerando como variable dependiente la Optimalidad e introduciendo todas las variables solicitadas

\begin{tabular}{|c|c|c|c|}
\hline \multirow{2}{*}{ Modelo } & $\begin{array}{c}\text { Variables } \\
\text { introducidas }\end{array}$ & $\begin{array}{c}\text { Variables } \\
\text { eliminadas }\end{array}$ & Método \\
\hline \multirow{2}{*}{1} & Disponibilidad & & \multirow{2}{*}{ Introducir } \\
\cline { 2 - 2 } & Afinidad & & \\
\cline { 2 - 2 } & Tamaño Inst. & & \\
\hline
\end{tabular}

\begin{tabular}{|c|c|c|c|c|c|c|}
\hline \multicolumn{7}{|c|}{ Coeficientes considerando como variable dependiente la optimalidad } \\
\hline & & \multicolumn{2}{|c|}{ Coeficientes no estandarizados } & \multirow{2}{*}{$\begin{array}{c}\text { Coeficientes } \\
\text { tipificados }\end{array}$} & \multirow[b]{2}{*}{$\mathrm{t}$} & \multirow[b]{2}{*}{ Sig. } \\
\hline Modelo & & $\mathrm{B}$ & Error típico & & & \\
\hline \multirow{4}{*}{1} & (Constante) & 0.958 & 0.023 & & 42.51 & 0 \\
\hline & Tamañolnst. & -0.065 & 0.006 & -0.789 & -10.3 & 0 \\
\hline & Afinidad & 0.04 & 0.006 & 0.486 & 6.347 & 0 \\
\hline & Disponibilidad & 0.007 & 0.006 & 0.085 & 1.109 & 0.279 \\
\hline
\end{tabular}

De acuerdo a los resultados obtenidos se puede apreciar que al comprobarse las Hipótesis $\mathrm{H} 1$ a H3, el tamaño de la instancia indica ser el parámetro que mayor significancia tiene en cuanto al tiempo de solución requerido para alcanzar la mejor solución posible. Así mismo, al observar los resultados de $\mathrm{H} 4$ a $\mathrm{H} 6$ y de $\mathrm{H} 7$ a $\mathrm{H} 9$ se puede apreciar un comportamiento similar entre ambos grupos respecto a que en ambos casos el valor de $t$ calculado sugiere un comportamiento parabólico al comparar los resultados del estudio de medias aritméticas implicando que el escenario del nivel medio es el que afecta negativamente el desempeño de la metaheurística aplicada. Esto podría explicarse ya que al aplicarse el nivel medio de estímulo el espacio de solución deja de estar restringido facilitando la búsqueda de la solución; sin embargo, al variar la afinidad hacia arriba, dificulta su exploración al incrementarse el espacio de solución a explorar en que se buscará la solución a alcanzar.

Conforme a los resultados aquí presentados se puede comentar que: El desempeño método de solución mediante algoritmos genéticos en este tipo de problemas se ve afectado en primer lugar por el tamaño de la instancia. Dicha aseveración se ve respaldada por el comportamiento tanto de la aproximación por parte de las pruebas de diferencias de medias (Pruebas t) así como de los resultados de los análisis de regresión. Esta 
situación era de esperarse debido a que: ante una mayor cantidad de opciones a combinar, el espacio de soluciones se amplía. En este caso en particular al incrementar al doble cada una de las dimensiones del problema representado por las instancias, el crecimiento del tamaño es exponencial recordando que se incrementa por un factor de 16 entre cada nivel sucesivo superior de este parámetro, teniendo como consecuencia una mucho mayor cantidad de combinaciones candidatas a ser exploradas por lo que tanto en términos de tiempo computacional como en optimalidad de la solución se presenta un impacto altamente significativo por parte de este parámetro (Tamaño de la instancia).

Con respecto al parámetro de afinidad de los docentes con las materias, en el caso de las pruebas de hipótesis, las evidencias indican que el nivel medio de la afinidad beneficia la exploración del espacio de soluciones ya que permite obtener en promedio un mejor resultado que cuando se evalúo en los escenarios Bajo y Alto; un comportamiento similar se encuentra en el parámetro de disponibilidad del docente. De lo anterior se puede deducir que, si bien los escenarios con estímulo Bajo tienen como consecuencia un espacio de soluciones más limitado, el método de solución no tiene una diversificación suficiente para explorarlo de una mejor manera en busca de óptimos; y por el contrario cuando se tiene un estímulo Alto, aunque se posee una mayor flexibilidad para diversificar la exploración el espacio de soluciones se incrementó de tal manera que su exploración se complica derivando, por lo tanto, en un mayor tiempo para que el método de solución converja en una respuesta. Los resultados obtenidos mediante los análisis de regresión nos permiten comprobar que: en el caso de estudiar el tiempo necesario para alcanzar una solución, el único parámetro significativo es el tamaño de la instancia; esta afirmación nos sugiere el que al incrementarse la extensión del espacio de soluciones la cantidad de combinaciones posibles se incrementa de una manera exponencial, comprobándose por lo que el método de solución requiere mayor tiempo para explorarlo; mientras que si el enfoque es analizar la optimalidad alcanzada se encontró que tanto el tamaño de la instancia como la afinidad de los docentes son los parámetros significativos al explicar una parte importante de la varianza en la variable explicada, lo cual apunta a que una mayor afinidad de los docentes permite tener mayor cantidad de opciones para diversificar la búsqueda y alcanzar una mayor cantidad de soluciones con alto puntaje.

\section{DISCUSIÓN Y ANÁLISIS FINAL}

De acuerdo a los hallazgos realizados durante la presente investigación, se puede declarar como comprobado para los escenarios estudiados, el efecto que los parámetros de la instancia tienen en las posibles soluciones a encontrar utilizando la metaheurística de Algoritmos Genéticos y presentar las siguientes afirmaciones:

El tamaño de la instancia es el parámetro que tiene un mayor efecto sobre el desempeño del método de solución estudiado. Un nivel medio de estímulo en los parámetros: a) Afinidad de los docentes con las materias y b) Disponibilidad horaria de los mismos beneficia la exploración del espacio de soluciones.

El tamaño de la instancia es el único parámetro significativo en cuando al tiempo de solución; no así en cuanto a la calidad de la solución obtenida, ya que en ese caso tanto el tamaño de la instancia como la afinidad de los docentes con las asignaturas se distinguieron como significativos para explicar la varianza de la variable dependiente. Los hallazgos obtenidos en el presente estudio complementan a otros trabajos que abordan la solución de problemas del tipo de calendarización de horarios desde un enfoque computacional y matemático, más orientados a las ciencias computacionales que a las sociales (Lewis y Thompson, 2015; Marín-Ángel y Maya Duque, 2016; Soria-Alcaraz et al. 2016).

\section{CONCLUSIONES}

De acuerdo al objetivo establecido del estudio, se puede concluir que el método de solución mediante algoritmos genéticos representa una alternativa conveniente para la resolución de problemas de asignación de recursos académicos debido a que la evaluación realizada demuestra que, mediante su aplicación, es posible obtener una solución de buena calidad en un tiempo aplicable; ya que aunque se comprobó que el tamaño de la instancia afecta proporcionalmente el tiempo de solución requerido, la mayor afinidad de docentes con respecto a las materias a impartir facilita el encontrar esas buenas soluciones. Por lo anterior el presente trabajo de investigación realiza una aportación al proceso de identificación de contextos para problemas de administración académica procurando apoyar a la tipificación de las características principales de una instancia a resolver y la ponderación de ellas al momento de elegir un método de solución para no verse limitado a utilizar métodos exactos y teniendo en consideración los efectos que ocasionarán las variaciones en los parámetros de la instancia a resolver mediante algún método metaheurístico no exacto.

\section{AGRADECIMIENTOS}

Los autores agradecen al Consejo Nacional de Ciencia y Tecnología de México (CONACYT) y a la Facultad de contaduría pública y administración de la Universidad autónoma de Nuevo León por el apoyo brindado para la realización del presente trabajo y reporte de investigación. 


\section{REFERENCIAS}

Ahumada, J. Á. y N. Balioan, Generación de horarios académicos en INACAP utilizando algoritmos genéticos, Santiago de Chile (2014)

Bellio, R., S. Ceschia y otros tres autores, Feature-based Tuning of Simulated Annealing Applied to the Curriculum-based Course Timetabling Problem, doi:10.1016/j.cor.2015.07.002, Computers and Operations Research, 65, 83-92 (2016)

Burke, E.K., J. Mareček, A.J. Parkes y H. Rudová, Decomposition, Reformulation, and Diving in University Course Timetabling, doi:10.1016/j.cor.2009.02.023, Computers and Operations Research, 37(3), 582-597 (2010)

Cancino, V.E. y T.S. Márquez, Evaluación de Desempeño de la Función Académica: Análisis de un Sistema en el Contexto Universitario Chileno, doi:10.4067/S0718-50062015000300005, Formación Universitaria, 8(3), 35-46 (2015)

Cataldo, A., J-C. Ferrer y otros tres autores, An Integer Programming Approach to Curriculum-based Examination Timetabling, doi:10.1007/s10479-016-2321-2, Annals of Operations Research, 258(2), 369-393 (2017)

Cortez, A., G. Rosales, R. Naupuri y H. Vega, Sistema de Apoyo a la Generación de Horarios basado en Algoritmos Genéticos, Revista de Investigación de Sistemas e Informática, 37-55 (2010)

Costabel, S., Metaheurísticas Aplicadas a un Problema de Asignación de salones y Horarios a Asignaturas, Montevideo, Universidad de la República (2005)

Dorigo, M. y T. Stützle, Ant Colony Optimization: Overview and Recent Advances, Handbook of Metaheuristics, Springer, Cham, 311-351 (2019)

Fong, C. W., H. Asmuni y otros tres autores, A new Hybrid Imperialist Swarm-based Optimization Algorithm for University Timetabling Problems, Information Sciences, 283, 1-21 (2014)

Goh, S. L., G. Kendall y N. R. Sabar, Improved Local Search Approaches to Solve the Post Enrolment Course Timetabling Problem, doi:10.1016/j.ejor.2017.01.040, European Journal of Operational Research, 261(1), 17-29 (2017)

Gómez-Garay, V., F. Artaza, E. Irigoyen y N. Iriondo, Un Método para la Asignación de la Docencia en el Ámbito Universitario Mediante Algoritmos Genéticos, Universidad del País Vasco, Bilbao (2005)

Grunitzki, R., G. de Oliveira Ramos y A. L. C. Bazzan, Individual Versus Difference Rewards on Reinforcement Learning for Route Choice, 2014 Brazilian Conference on Intelligent Systems, IEEE, 253-258 (2014)

Holland, J. H., Adaptation in Natural and Artificial Systems: an Introductory Analysis with Applications to Biology, Control, and Artificial Intelligence, $1^{\text {a }}$ Ed., 1-14. MIT press, Cambridge, Mass (1992)

Johnston Barrientos, J. D., Aplicación de Algoritmos Genéticos para la Asignación de Carga Académica en Instituciones de Educación Superior, Disertación Doctoral, Universidad Autónoma de Nuevo León (2000)

Lewis, R. y J. Thompson, Analysing the Effects of Solution Space Connectivity with an Effective Metaheuristic for the Course Timetabling Problem, doi:10.1016/j.ejor.2014.07.041, European Journal of Operational Research, 240(3), 637$648(2015)$

López, B. y J. D. Johnston, Modelo de Asignación de Carga Académica usando Algoritmos Genéticos (2007)

Marín-Ángel, J.C. y P.A. Maya-Duque, Modelo Lineal para la Programación de Clases en una Institución Educativa, doi:10.17230/ingciencia.12.23.3, Ingeniería y Ciencia, 12(23), 47-71 (2016)

RAE., Real Academia Española - Heurística (2012)

Ramos, G. D. O. y A. L. C. Bazzan, Towards the User Equilibrium in Traffic Assignment using GRASP with Path Relinking, Proceedings of the 2015 Annual Conference on Genetic and Evolutionary Computation, ACM, 473-480 (2012)

Sanchez-Partida, D., E. G. Baquela, J. Mora-Vargas y N. R. Smith, Case study: Simulated Annealing for Improving the Educational Timetable, doi: 10.21640/ns.v8i17.631, Nova Scientia, ISSN: 2007-0705, 8(17), 340-360 (2016)

Soria-Alcaraz, J.A., E. Özcan y otros tres autores, Iterated Local Search using an Add and Delete Hyper-heuristic for University Course Timetabling, doi:10.1016/j.asoc.2015.11.043, Applied Soft Computing, 40, 581-593 (2016)

Tsutsui, S. y N. Fujimoto, Fast QAP Aolver with ACO and Taboo Search on GPU using Move-cost Adjusted Thread Assignment, Genetic and Evolutionary Computation Conference, 1-2 (2011)

Vannucci, J., D. Garrett y D. Dasgupta, Comparative Analysis of the Sailor Assignment Problem, Proceedings of the 8th Annual Conference on Genetic and Evolutionary Computation, ACM, 1881-1882 (2006)

Vásquez, A. C., H. V. Huerta, G. R. Gerónimo y R. N. Quiroz, Sistema de Apoyo a la Generación de Horarios basado en Algoritmos Genéticos, Revista de investigación de Sistemas e Informática, 7(1), 37-56 (2010)

Vergel miranda, A., Algoritmo para la Asignación de Horarios Académicos en la Universidad Francisco de Paula Santander Ocaña utilizando Técnicas de Inteligencia Artificial, Disertación Doctoral, Universidad Francisco de Paula Santander Ocaña (2017)

Zuniga-Jara, S., J. Barraza-Carvajal, N. Sanhueza-Muñoz y S. Soza-Amigo, Midiendo la Creación de Valor en una Universidad, doi:10.4067/S0718-50062018000300087, Formación Universitaria, 11(3), 87-96 (2016) 